\title{
In Vitro Expression Demonstrates Impaired Secretion of the $\gamma$ Asn319, Asp320 Deletion Variant Fibrinogen
}

\section{Satomi Kani ${ }^{1}$, Fumiko Terasawa ${ }^{2}$, Susan T Lord ${ }^{3}$, Minoru Tozuka ${ }^{1}$, Hiroyoshi Ota², Nobuo Okumura², Tsutomu Katsuyama ${ }^{1}$}

1) Department of Laboratory Medicine and 2) Department of Biomedical Laboratory Sciences, Shinshu University School of Medicine, Matsumoto Japan and 3) Department of Pathology and Laboratory Medicine, University of North Carolina, Chapel Hill, NC, USA.

Word count: 4086, (Summary word count: 238)

Correspondence to: Nobuo Okumura, $\mathrm{PhD}$

Department of Biomedical Laboratory Sciences, School of Health Sciences, Shinshu University, 3-1-1 Asahi, Matsumoto 390-8621, Japan

Phone:(81) 26337 2392; Fax: (81) 26337 2370;

E-mail: nobuoku@gipac.shinshu-u.ac.jp

A short running title: Impaired Fibrinogen Secretion of the $\gamma \Delta \mathrm{N} 319, \mathrm{D} 320$ 


\section{Summary}

The hypodysfibrinogenemia Otsu is caused by the two-residue deletion, $\gamma$ Asn319 and $\gamma$ Asp320. Analysis of plasma or purified fibrinogen from the heterozygous propositus revealed that the amount of variant $\gamma$-chain was lower than that of normal $\gamma$-chain. In order to examine the basis for this difference, we transfected Chinese hamster ovary cells and established stable cell lines that expressed both chains, $\gamma \Delta / \gamma \mathrm{N}$, only the normal chain, $\gamma \mathrm{N}$, and only the variant chain, $\gamma \Delta$. We measured fibrinogen concentration of confluent cultures by ELISA. We found the ratios of the concentrations in the media to the concentrations in the cell lysates of $\gamma \Delta, \gamma \Delta / \gamma \mathrm{N}$, and $\gamma \mathrm{N}$-cells were $0.42,0.60$, and 1.00 , respectively. We measured the concentrations of the $\gamma \Delta$ and $\gamma \mathrm{N}$ chains by densitometric analysis of samples following separation by SDS-PAGE and found the fraction of $\gamma \Delta$-chains in cell lysates was always greater than the fraction in the respective culture media. We examined the kinetics of fibrinogen synthesis, assembly and secretion in pulse-chase experiments, and found that the $\gamma \Delta$-chain was assembled into intact fibrinogen at a rate similar to assembly of the $\gamma \mathrm{N}$-chain into normal fibrinogen, but was secreted into the medium at a slightly slower rate than normal fibrinogen. Considered together, these experiments indicate secretion of the variant fibrinogen was slightly impaired. These results suggest that the reduced level of $\gamma \Delta 319,320$ fibrinogen in the plasma of the Otsu patient arises from modestly impaired secretion of this variant fibrinogen.

Key Words: assembly, dysfibrinogen, pulse-chase analysis, secretion, $\gamma$-chain 


\section{Introduction}

Fibrinogen is a $340 \mathrm{kDa}$ dimeric plasma glycoprotein that circulates in the blood at 1.8 $3.5 \mathrm{~g} / \mathrm{L}$. It is composed of two copies of each of three polypeptide chains, $A \alpha, B \beta$ and $\gamma$, which are linked by 29 intra- and inter-chain disulfide bonds [1]. The fibrinogen molecule has a trinodular structure where the central nodule contains the N-termini of all six chains and the external nodules contain the C-termini of the B $\beta$ - and $\gamma$-chains [2]. The nodules are linked by two coiled coils, consisting of all three chains [2]. The chains are synthesized, assembled into a 6-chain glycosylated molecule, and secreted from hepatocytes into the blood. The synthesis, assembly and secretion of fibrinogen has been examined by pulse-chase experiments in cultured human hepatoma cells, HepG2, and by analysis of intermediates expressed from recombinant vectors in cultured mammalian cells [3-6]. These experiments show assembly is a multistage event, which includes specific intermediates such as $A \alpha-\gamma$ and $B \beta-\gamma$ complexes, and does not depend on hepatic specific factors [4,5]. Further analyses of recombinant variant fibrinogens with substitutions or deletions have identified residues and domains that are and are not required for assembly and secretion [6]. These studies have demonstrated, for example, that the $\mathrm{N}$-terminal domains of each chain are critical for dimer assembly, that the C-terminal half of the coiled-coil and intrachain disulfides proximate to the coiled-coil are critical for formation of the initial two chain complexes, that the C-terminal intrachain disulfide in the $\gamma$-chain is critical for secretion but not intracellular assembly, and that the C-terminal intrachain disulfides in the A $\alpha$ and $\mathrm{B} \beta$-chains are not critical for either assembly or secretion [6]. Recent studies with truncated variants have shown that residues near the C-terminus of the $\gamma$-chain, in particular rIle387, are also critical for formation of the initial two chain complexes [7]. Thus, it has been shown repeatedly that the $\mathrm{C}$-terminal region of the $\gamma$-chain has an important role in intact fibrinogen biosynthesis.

Studies of familial dysfunctional fibrinogens have identified several cases associated with reduced levels of total circulating fibrinogen due to reduced levels of the defective fibrinogen [8]. Analysis of the synthesis and secretion of the variant fibrinogens in these patients has not been possible, as access to liver tissue is limited. Missense mutations have also been reported in 6 families with hypofibrinogenemia and 4 families with afibrinogenemia, where fibrinogen levels are reduced and absent, respectively [9-17]. In these cases the synthesis, assembly and/or secretion of the variant fibrinogens is presumably impaired. Indeed, in two cases with missense 
mutations in the $\gamma$-chain, the variant fibrinogens cause hepatic endoplasmic reticulum storage disease $[11,12]$. We have examined the synthesis and secretion of the dysfibrinogenemia caused by the substitution of $\gamma 153 \mathrm{Cys}$ to Arg, and found that assembly and secretion of this variant fibrinogen in Chinese Hamster Ovary (CHO) cells was impaired [10]. Considering both the data from patients and the results from in vitro expression, we believe the molecular basis for at least some hypofibrinogenemias and afibrinogenemias is disruption of the tertiary structure of this C-terminal $\gamma$-chain domain.

Recently we identified a hypodysfibrinogenemia, termed fibrinogen Otsu [18], caused by the deletion of $\gamma \operatorname{Asn} 319$ and $\gamma \operatorname{Asp} 320(\gamma \Delta 319,320)$, the same deletion found in the Vlissingen/Frankfurt-IV dysfibrinogenemia [19]. Interestingly, we found that the amount of variant $\gamma$-chain, which we call $\gamma \Delta$, in the patient's plasma was lower than the normal $\gamma$-chain. Similar findings were seen in a mouse model of this dysfibrinogenemia [26]. Hogan et al. used gene targeting to generate mice with the comparable deletion in the mouse $\gamma$-chain gene. Mice that were heterozygous for this deletion had about one half the level of fibrinogen as normal mice, while mice homozygous for the deletion had about one eighth the normal level [26]. These date suggest that the amount of the variant $\gamma \Delta$-chain in the fibrinogen of heterozygous mice was lower than the amount the normal $\gamma$-chain, as was found in the Otsu patient. Analysis of gene expression in livers of heterozygous mice by quantitative rt-PCR showed that expression of $\gamma \Delta$-mRNA was not different from expression of $\gamma$-mRNA, indicating impaired assembly and secretion of this variant fibrinogen in mice. Hogan et al. have also synthesized and characterized recombinant $\gamma \Delta 319,320$ fibrinogen [20]. They found that this deletion affected essentially all functions associated with the $\mathrm{C}$-terminal domain of the $\gamma$-chain, suggesting a marked disruption of the overall tertiary structure of the $\mathrm{C}$-terminal domain of the variant chain [20]. Based on these data, we hypothesized that the reduced levels of $\gamma \Delta 319,320$ fibrinogen in humans and mice might be due to decreased synthesis and secretion associated with a disruption in the tertiary structure of this domain. To test this hypothesis, we examined the rate of synthesis and/or secretion of this variant fibrinogen in cultured $\mathrm{CHO}$ cells.

\section{Materials and Methods}

Purification of plasma fibrinogens. Plasma fibrinogens from the normal control and the propositus of Matsumoto-II ( $\gamma 308 \mathrm{Asn}->$ Lys, $\gamma \mathrm{N} 308 \mathrm{~K})$ [21] and Otsu [18] were purified by a 
modified immunoaffinity chromatography procedure using an IF-1 monoclonal antifibrinogen [22]. Eluted fibrinogen fractions were pooled and dialyzed against 20mM N-(2-hydroxyethyl) piperazine-2'-(2-ethanesulfonic acid) (HEPES), pH 7.4 containing 0.12M NaCl. Fibrinogen concentrations were determined from the $\Delta \mathrm{A}_{280-320}$ value [23].

Recombinant protein expression. The variant $\mathrm{pMLP}-\gamma$ vector $(\mathrm{pMLP}-\gamma \Delta)$ was generated by oligonucleotide-directed mutagenesis as described elsewhere [24] and pMLP- $\gamma \Delta$ and/or original pMLP- $\gamma$ vector were cotransfected with the histidinol selection plasmid (pMSVhis) into the $\mathrm{CHO}-\mathrm{A} \alpha \mathrm{B} \beta$ cell line expressing normal human fibrinogen $\mathrm{A} \alpha$ - and $\mathrm{B} \beta$-chains, using the standard calcium-phosphate coprecipitation method [25]. Cells were cultured in Dulbecco's modified Eagle medium Ham's nutrient mixture F12 (DMEM-F12 medium), and colonies were selected on both G418 (GIBCO BRL, Rockvill, MD, USA) and histidinol (Aldrich Chem. Co. Milwaukee, WI, USA) [25].

Immunoassays. Sodium dodecyl sulfate-polyacrylamide gel electrophoresis (SDS-PAGE) and immunoblot analysis was performed as described with minor modifications [7,10,25]. Immunoblots were developed with a rabbit antihuman fibrinogen antibody (DAKO, Carpinteria, CA, USA) and reacting species were visualized with horseradish peroxidase-conjugated goat antirabbit IgG antibody (Medical and Biological Laboratories Ltd, Nagoya, Japan) and enhanced chemiluminescence (ECL) detection reagents (Amersham Pharmacia Biotech Inc., Buckinghamshire, United Kingdom) [7]. Alternatively blots were developed with a mouse antihuman fibrinogen $\gamma$-chain monoclonal antibody (4A5, Boston Research Services Company, Wellesley, MA), followed by horseradish peroxidase-conjugated goat antimouse IgG antibody (Medical and Biological Laboratories, Ltd), and developed as described above. Fibrinogen concentrations in cell lysates or culture media were determined by enzyme-linked immunosorbent assay (ELISA), as described [10]. Statistical values comparing normal and variant recombinant fibrinogens were determined using the unpaired $t$-test and a difference was significant when the $p$ was $<0.05$.

Culture medium or cell lysates for immunologic analysis were prepared as follows. Cells were grown to confluence in 60-mm dishes (approximately $1.5-2.0 \times 10^{6}$ cells), and the conditioned medium was harvested one day post confluence ( 6 to 8 days after seeding) for immunoblot 
analysis or ELISA. Cell lysates were prepared from the same cultures. The cells were harvested in trypsin-EDTA solution (Sigma, St Louis, MO), washed three times with phosphate-buffered saline (PBS), and lysed in either $50 \mu \mathrm{L}$ of Laemmli sample buffer for immunoblot analysis, or $250 \mu \mathrm{L}$ of $0.1 \%$ IGEPAL CA-630 (Sigma) and $10 \mathrm{mmol} / \mathrm{L}$ phenylmethylsulfonyl fluoride (Sigma) for ELISA.

Pulse-chase analysis of protein synthesis using $\left[{ }^{35} \mathrm{~S}\right]$ methionine. Pulse-chase studies were performed as described [10] for selected cell lines, $\gamma \mathrm{N}-21, \gamma \Delta-34$ and $\gamma \Delta / \gamma \mathrm{N}-11$. In brief, the medium was replaced with $1 \mathrm{~mL}$ of methionine-free DMEM supplemented with $1.5 \mathrm{MBq} \mathrm{L}-$ $\left[{ }^{35} \mathrm{~S}\right]$ methionine (Amersham Pharmacia Biotech Inc) and the cells were incubated in $5 \% \mathrm{CO}_{2}$ for 60 minutes at $37^{\circ} \mathrm{C}$. After the pulse, the cells were rinsed twice, $1 \mathrm{~mL}$ of fresh DMEM containing $20 \mathrm{mM}$ unlabeled L-methionine (Wako, Osaka, Japan) was added and the cells were incubated for various chase periods. Culture media or cell lysates were added to an equivalent amount of 1:1000 diluted rabbit anti-fibrinogen polyclonal antibody (DAKO) and incubated overnight at $4{ }^{\circ} \mathrm{C}$ and immunocomplexes precipitated with Protein A-Sepharose. The precipitates dissolved with Laemmli sample buffer were resolved on SDS-polyacylamide gradient gels. Radioactive bands were detected with the Fujix Bio-Imaging Analyzer BAS2000 System (Fuji Photo Film Co, Tokyo, Japan).

\section{RESULTS}

Characterization of fibrinogen Otsu.

Immunoblot analysis of plasma from the Otsu propositus [18] demonstrated that the $\gamma \Delta$-chain migrated faster than the normal $\gamma$-chain, and that the staining intensity of the variant band with either a rabbit antihuman fibrinogen polyclonal antibody or a mouse antihuman fibrinogen $\gamma$-chain monoclonal antibody was less than normal band (Fig 1A,B). This contrasted with similar analysis of another $\gamma$-chain dysfibrinogen, Matsumoto II (M-II), where the staining intensities for the variant $\gamma$-chain and the normal $\gamma$-chain were similar (Fig 1A,B). Further, analysis of purified fibrinogen from the Otsu propositus showed a similar disparity with Coomassie staining (Fig 1C) and densitometric analysis indicates the variant band was $51 \%$ of the normal band. Again, this differed from fibrinogen purified from the M-II propositus where the variant band was $106 \%$ of the normal band (Fig 1C). We conclude that in the Otsu patient's fibrinogen, the amount of variant $\gamma$-chain was less than that of the normal $\gamma$-chain. 
Synthesis and secretion of the variant fibrinogen in CHO cells.

The mutant expression vector, pMLP- $\gamma \Delta$, was previously generated by oligonucleotide-directed mutagenesis of the normal $\gamma$-chain expression vector, pMLP- $\gamma$ [24]. We cotransfected pMLP- $\gamma$ and/or pMLP- $\gamma \Delta$ with pMSVhis into the CHO-A $\alpha \mathrm{B} \beta$ cell line [7]. From each transfection twenty to thirty histidinol resistant clones secreting fibrinogen were identified by ELISA of the culture media. We selected 4 clones of $\gamma \mathrm{N}-\mathrm{CHO}$ cells, which synthesized normal fibrinogen, and 4 clones of $\gamma \Delta$-CHO cells, which synthesized the variant $\gamma \Delta 319,320$ fibrinogen, based on the similar fibrinogen concentrations in cell lysates. CHO cells trasfected with both normal and variant chains were also screened by immunoblot analysis of cell lysates. We selected 6 clones of $\gamma \Delta / \gamma \mathrm{N}-\mathrm{CHO}$ cells with both normal and variant chains in cell lysates. Finally, we selected three cell lines, clones $\gamma \mathrm{N} 21-, \gamma \Delta 34-$, and $\gamma \Delta / \gamma \mathrm{N} 11-\mathrm{CHO}$, to use in pulse-chase and time course analysis of fibrinogen synthesis and secretion, again based on the similar fibrinogen concentrations in cell lysates.

Fibrinogen concentrations were determined by ELISA of the culture media and cell lysates that were harvested one day following cell confluence. The results are presented in Table 1. Analysis of the $\mathrm{CHO}-\mathrm{A} \alpha \mathrm{B} \beta$ cell line used for these transfections demonstrated that $<0.01 \mu \mathrm{g} / \mathrm{mL}$ fibrinogen was present in either the culture media or the cell lysates as previously described [10]. Comparing the data from either several cultures or individual clones showed the concentration of variant fibrinogen in the medium was significantly lower than that in the cell lysates relative to the respective concentrations for normal fibrinogen. For example, the ratio of fibrinogen concentration in the medium to fibrinogen concentration in the cell lysates averaged from 4 lines expressing normal fibrinogen was $1.0 \pm 0.1$, while for 4 lines expressing the $\gamma \Delta 319,320$ variant this ratio was $0.42 \pm 0.07$. 
Table 1. Fibrinogen concentration of media and cell lysates from $\mathrm{CHO}$ cell lines

\begin{tabular}{lccc}
\hline $\begin{array}{c}\text { CHO cell lines }(\mathrm{n}) \\
\text { or selected clone \# }\end{array}$ & $\begin{array}{c}\text { media } \\
(\mu \mathrm{g} / \mathrm{ml})\end{array}$ & $\begin{array}{c}\text { cell lysates } \\
(\mu \mathrm{g} / \mathrm{ml})\end{array}$ & $\begin{array}{c}\text { medium/cell lysate } \\
\gamma \mathrm{N}(4)^{1}\end{array}$ \\
$1.8 \pm 0.7$ & $1.9 \pm 0.7$ & $1.00 \pm 0.12$ \\
$\gamma \Delta(4)^{1}$ & $0.67 \pm 0.12$ & $1.6 \pm 0.3$ & $0.42 \pm 0.07^{\mathrm{a}}$ \\
$\gamma \Delta / \gamma \mathrm{N}(6)^{1}$ & $1.9 \pm 0.7$ & $3.1 \pm 0.8$ & $0.60 \pm 0.06^{\mathrm{b}}$ \\
$\gamma \mathrm{N} 21^{2}$ & $2.4 \pm 0.2$ & $2.3 \pm 0.2$ & $1.04 \pm 0.11$ \\
$\gamma \Delta 34^{2}$ & $0.64 \pm 0.05$ & $1.8 \pm 0.3$ & $0.36 \pm 0.04^{\mathrm{c}}$ \\
$\gamma \Delta / \gamma \mathrm{N} 11^{2}$ & $2.1 \pm 0.5$ & $2.5 \pm 0.3$ & $0.84 \pm 0.13$ \\
\hline
\end{tabular}

${ }^{1}$ Averages from three or more determinations of multiple cell lines.

${ }^{2}$ Averages from three determinations of a single cell line.

${ }^{a}$ Significantly different from the $\gamma \mathrm{N}$ cell lines $(p<0.01)$.

${ }^{\mathrm{b}}$ Significantly different from the $\gamma \mathrm{N}$ cell lines $(p<0.02)$.

'Significantly different from the $\gamma \mathrm{N} 21$ cell line $(p<0.01)$

Immunoblot analysis of cell lysates for the selected clones $\gamma \mathrm{N} 21$ and $\gamma \Delta 34$ and six clones of $\gamma \Delta / \gamma \mathrm{N}-\mathrm{CHO}$ showed several reacting bands including intact fibrinogen and the individual chains in all cases (Fig 2A), when gels were run under nonreducing conditions. The lysates of the $\gamma \Delta / \gamma \mathrm{N}$-CHO cells showed a broad $\gamma$-chain band (Fig 2A) Similar immunoblots of gels run under reducing conditions showed $\mathrm{A} \alpha-, \mathrm{B} \beta-$, and $\gamma$-chains as well as several smaller species, presumably proteolytic degradation products, in all cell lysates (Fig 2B). Lysates of the $\gamma \Delta / \gamma \mathrm{N}$-CHO cells showed two distinct bands with various ratios of intensities for the $\gamma \Delta$-chains and normal $\gamma \mathrm{N}$-chains (Fig 2B and Table2), suggesting variable synthesis of the $\gamma \Delta$ - and $\gamma \mathrm{N}$-chains. Immunoblot analysis of the media from similar cultures of $\gamma \mathrm{N}-, \gamma \Delta-$, and $\gamma \Delta / \gamma \mathrm{N}-\mathrm{CHO}$ cells showed the presence of fibrinogen or A $\alpha-$, B $\beta$-, and $\gamma$-chains under nonreducing (Fig 2C) or reducing conditions (Fig 2D), respectively. Analysis of the media from the $\gamma \Delta / \gamma \mathrm{N}-\mathrm{CHO}$ cells showed the presence of both $\gamma \Delta$ - and $\gamma \mathrm{N}$-chains, demonstrating that both chains were assembled into fibrinogen and secreted into the medium (Table2). In every 
case the fraction of $\gamma$-chain in the medium exceeded that in the cell lysates, with a mean of $1.37 \pm$ 0.21 -fold $(p<0.02)$ increase. These data suggest that assembly and/or secretion of the $\gamma \Delta$-chain was impaired relative to the $\gamma \mathrm{N}$-chain (Table2).

Table 2 Densitometoric analysis for $\gamma \Delta$ - and $\gamma \mathrm{N}$ - chains in cell lysates and media of $\gamma \Delta / \gamma \mathrm{N}-\mathrm{CHO}$ cell lines

\begin{tabular}{|l|c|c|c|c|c|c|c|}
\hline Clone numbers & 4 & 11 & 20 & 35 & 41 & 56 & Mean \pm SD \\
\hline $\begin{array}{l}\gamma \mathrm{N} /(\gamma \Delta+\gamma \mathrm{N}) \text { in cell } \\
\text { lysates x } 100 \%\end{array}$ & $56 \%$ & $28 \%$ & $56 \%$ & $66 \%$ & $69 \%$ & $70 \%$ & $57.5 \pm 15.7 \%$ \\
\hline $\begin{array}{l}\gamma \mathrm{N} /(\gamma \Delta+\gamma \mathrm{N}) \text { in } \\
\text { media x } 100 \%\end{array}$ & $85 \%$ & $48 \%$ & $67 \%$ & $77 \%$ & $87 \%$ & $89 \%$ & $75.5 \pm 15.7 \%$ \\
\hline $\begin{array}{l}\% \gamma \mathrm{N} \text { in media/ } \\
\% \gamma \mathrm{N} \text { in cell lysates }\end{array}$ & 1.52 & 1.71 & 1.26 & 1.17 & 1.26 & 1.27 & $1.37 \pm 0.21^{*}$ \\
\hline
\end{tabular}

$* P<0.02$ unpaired t-test for 1.00 .

Pulse-chase analysis of fibrinogen synthesis.

We performed pulse-chase experiments to examine the assembly and secretion of normal and variant fibrinogens using $\gamma \mathrm{N} 21-, \gamma \Delta 34-$, and $\gamma \Delta / \gamma \mathrm{N} 11-\mathrm{CHO}$ cells. Cells were labeled with $\left[{ }^{35} \mathrm{~S}\right]$-methionine and chased with unlabeled methionine for up to 24 hours. Radiolabeled fibrinogen was immunoprecipitated from cell lysates and culture media and the precipitates were analyzed by SDS-PAGE, as shown in Fig 3. The specificity of the immunoprecipitation was verified by the loss of labeled bands when excess plasma fibrinogen was added to samples prior to immunoprecipitation (Fig 3A, lane Inh).

Analysis of lysates from $\gamma \mathrm{N}-\mathrm{CHO}$ cells showed an immunospecific band at $340 \mathrm{kDa}$, as expected for the intact fibrinogen molecule (Fig 3A) $[3,4]$. This band was clearly present prior to the chase, increased in intensity for 2-3 hours into the chase and decreased thereafter, indicating that fibrinogen was stable within the cell for several hours. Similar results are seen with lysates from $\gamma \Delta$-CHO and $\gamma \Delta / \gamma \mathrm{N}-\mathrm{CHO}$ cells; the $340 \mathrm{kDa}$ band was apparent prior to the chase, the intensity of this band increased for 2-3 hours during the chase and decreased thereafter (Fig 
$3 \mathrm{~B}, \mathrm{C})$. The intensity of the $340 \mathrm{kDa}$ band prior to the chase was weaker in the $\gamma \Delta$-CHO lysates, indicating that the rate of fibrinogen synthesis or assembly was slightly slower in this cell line. This result may simply reflect the slightly lower expression of the $\gamma$-chain in this cell line (Table 1). Of note, the intensities of the individual chains and the bands that presumably represent assembly intermediates did not differ between the cell lines. Thus, there was no obvious delay in the formation of intermediates or the assembly of intermediates into fibrinogen. These data suggest that assembly of the variant fibrinogen was not significantly impaired relative to normal.

Analysis of the culture media showed that radiolabeled normal fibrinogen was first detectable in the media following the 2-hour chase (Fig 3A). Thereafter, the intensity increased with each time point, indicating that fibrinogen continued to be secreted throughout the course of this experiment. The increase in radiolabeled fibrinogen in the medium paralleled the decrease in radiolabeled fibrinogen in the cell lysates, as expected for normal secretion. The culture media from $\gamma \Delta$-CHO cells showed radiolabeled immunospecific bands in the 3-hour chase and the intensity of this band increased with time (Fig 3B). The results with $\gamma \Delta / \gamma \mathrm{N}-\mathrm{CHO}$ cells were intermediate with a weak radiolabeled immunospecific band seen following the 2-hour chase and increasing up to 24 hours (Fig 3C). These data suggest that the secretion of the variant fibrinogen was slightly impaired relative to normal.

\section{Time course of fibrinogen secretion from $\mathrm{CHO}$ cells.}

We determined the time course of fibrinogen secretion from eight $60-\mathrm{mm}$ cultures of each cell line. One day following confluence, fresh medium was added and cultures maintained for an additional 6 days. During these cultures, cell-death and cell-detach from dishes were not observed. Fibrinogen concentrations were determined in lysates of cells harvested at confluence (from 5 culture plates, at day 0 ) and the culture media from days 0 to 6 . The ratio of fibrinogen in the media to fibrinogen in the cell lysates at day 0 increased with culture time (Fig. 4). The data at days 1 and 6 are summarized in Table 3. After 6 days of culture the ratio of fibrinogen in the medium to that in the cell lysates at day 0 reached 2.35 for $\gamma \mathrm{N}-\mathrm{CHO}$ cells, 1.00 for $\gamma \Delta$-CHO cells and 1.95 for $\gamma \Delta / \gamma \mathrm{N}-\mathrm{CHO}$ cells. The fibrinogen concentrations in the variant cell lysates relative to $\gamma \mathrm{N}-\mathrm{CHO}$ cell lysates were: 0.65 for $\gamma \Delta-\mathrm{CHO}$ cells and 0.95 for $\gamma \Delta / \gamma \mathrm{N}-\mathrm{CHO}$ cells, while the relative concentrations in media were: 0.25 for $\gamma \Delta$-CHO cells and 0.64 for $\gamma \Delta / \gamma \mathrm{N}-\mathrm{CHO}$ cells at day 1 . In all cases (Table 3) the relative concentrations of the 
variant fibrinogens $(\gamma \Delta-\mathrm{CHO}$ and $\gamma \Delta / \gamma \mathrm{N}-\mathrm{CHO})$ in the media was lower that those in the cell lysates, indicating that secretion of the variant fibrinogen was impaired.

Table 3. Fibrinogen concentration $(\mu \mathrm{g} / \mathrm{ml})$ in culture media and cell lysates

\begin{tabular}{cccc}
\hline & \multicolumn{3}{c}{ CHO cell line } \\
\cline { 2 - 4 } measurement & $\gamma \Delta 34$ & $\gamma \Delta / \gamma \mathrm{N} 11$ & $\gamma \mathrm{N} 21$ \\
\hline cell lysates & $1.3 \pm 0.1$ & $1.9 \pm 0.1$ & $2.0 \pm 0.1$ \\
(at confluence) & $(0.65)$ & $(0.95)$ & $(1.00)$ \\
media & $0.55 \pm 0.04$ & $1.6 \pm 0.1$ & $2.0 \pm 0.1$ \\
(at confluence) & $(0.28)$ & $(0.80)$ & $(1.00)$ \\
media & $0.55 \pm 0.05^{\mathrm{b}}$ & $1.4 \pm 0.1$ & $2.2 \pm 0.1$ \\
(day1) $^{\mathrm{a}}$ & $(0.25)$ & $(0.64)$ & $(1.00)$ \\
Media & $1.3 \pm 0.1^{\mathrm{b}}$ & $3.7 \pm 0.2^{\mathrm{c}}$ & $4.7 \pm 0.1$ \\
(day6) $^{\mathrm{a}}$ & $(0.28)$ & $(0.79)$ & $(1.00)$
\end{tabular}

Fibrinogen concentrations are mean $\pm \mathrm{SD}$ of triplicate cultures. Figures in parenthesis indicate mean values relative to $\gamma \mathrm{N} 21$ - $\mathrm{CHO}$ cells.

a Media was removed from confluent cells, replaced with fresh media and cultured for 1 or 6 days.

${ }^{b}$ Significantly different from $\gamma \mathrm{N} 21$ cell line $(p<0.001)$.

${ }^{c}$ Significantly different from $\gamma \mathrm{N} 21$ cell line $(p<0.05)$.

\section{DISCUSSION}

The cellular mechanisms that control fibrinogen assembly and secretion have previously been studied in transfected mammalian cell cultures where the expressed chains were modified either to address the importance of specific chains, domains or residues, or to imitate mutations identified in hypo- or afibrinogenemias [3-7]. Here we examined fibrinogen assembly and secretion of a dysfibrinogenemia where the variant, $\gamma \Delta 319,320$ fibrinogen, is found in patient plasma, although at a reduced level relative to normal. Moreover, similar data were found in a mouse model of this dysfibrinogenemia, where the analogous two amino acid deletion was introduced into mice by gene targeting [26]. Mice heterozygous for this deletion appeared 
healthy, while homozygous mice suffered from neonatal bleeding. Plasma fibrinogen levels were reduced in both heterozygous and homozygous mice, to $50 \%$ and $12 \%$ respectively. These findings suggested that analysis of expression of $\gamma \Delta 319,320$ fibrinogen would provide insight into the mechanisms that control assembly and secretion. Our data indicate that synthesis and assembly of the variant fibrinogen are normal, while secretion of the variant is slightly impaired relative to secretion of normal fibrinogen.

We established and characterized multiple cell lines that expressed normal fibrinogen or $\gamma \Delta 319,320$ fibrinogen or a mixture of normal and $\gamma \Delta 319,320$ fibrinogens. Analysis of these lines clearly demonstrated that $\gamma \Delta 319,320$ fibrinogen was secreted into the medium, but at lower levels relative to its presence inside the cell. This finding was independent of whether $\gamma \Delta 319,320$ fibrinogen was expressed alone or in combination with normal fibrinogen. The pulse-chase analyses of the culture media and cell lysates, which would expose differences in the rates of assembly and secretion, indicated that for both the pure variant and the mixed molecules these rates were very similar to normal with a slight delay apparent in the appearance of the pure variant in the culture media. As we did not see accumulation of any assembly intermediate in the variant lines, we conclude that no specific step in assembly is markedly impaired. Nevertheless, the ratio of secreted to intracellular fibrinogen determined by ELISA of confluent cultures, or 1 or 6 day post confluent cultures showed that secretion of $\gamma \Delta$-fibrinogen was impaired relative to secretion of normal fibrinogen. These results suggest that secretion, rather than synthesis and/or assembly, was impaired. Because analysis of the cell lysates reflects the balance of fibrinogen synthesis, assembly, secretion, and intracellular degradation while analysis of the conditioned media reflects the balance of fibrinogen secretion and extracellular degradation, it is not possible to determine which step or steps cause the lower levels in the media. We believe it is unlikely that the variant per se would alter the rate of degradation in the media and we did not detect such degradation products (Fig 2D), so we conclude that the lower level of variant in the culture media reflects reduced secretion associated with intracellular degradation of the nonsecreted $\gamma \Delta$-fibrinogen in $\gamma \Delta-\mathrm{CHO}$ and $\gamma \Delta / \gamma \mathrm{N}-\mathrm{CHO}$ cells.

These data and this conclusion differ significantly from those of our previous experiments that examined assembly and secretion of variant fibrinogens. In one study we showed expression of the variant $\gamma$ Cys $153 \mathrm{Arg}$ chain in CHO cells was associated with impaired assembly and thereby 
secretion [10]. We concluded that the loss of Cys at residue 153 caused profound changes in the overall structure of the $\mathrm{C}$-terminal domain of the $\gamma$-chain, and thus impaired assembly. In another study we directly examined the role of the $\gamma$-chain, by monitoring synthesis of fibrinogens with increasing deletions from the $\mathrm{C}$-terminus of the $\gamma$-chain. We found that residues near the C-terminus of the $\gamma$-chain were essential for fibrinogen assembly, and more specifically, that $\gamma$ Ile387 was critical [7]. We concluded that the loss of $\gamma$ Ile387 markedly changed the overall tertiary structure of $\gamma$-chain, preventing assembly of $\alpha \gamma$ and $\beta \gamma$ dimers, which others have shown to be essential intermediates in the assembly of normal fibrinogen [4,5]. Thus, impaired assembly has been correlated with changes in the structure of the C-terminal domain of the $\gamma$-chain. Previous studies with recombinant $\gamma \Delta 319,320$ fibrinogen demonstrate that all functions associated with the C-terminal domain on the $\gamma$-chain were affected, suggesting a disruption of the tertiary structure of this C-terminal domain [20]. Nevertheless, this structural disruption did not prevent assembly of the $\gamma \Delta 319,320$ chain into fibrinogen. We conclude that either the tertiary structure required for assembly is not disrupted in $\gamma \Delta 319,320$ fibrinogen, or the prior correlation between the overall tertiary structure of this C-terminal domain and fibrinogen chain assembly is deceptive. More studies are needed to resolve this ambiguity.

Studies of hypo- and afibrinogenemias have demonstrated that the absence of variant chains in the plasma arises from impaired secretion of assembled fibrinogen. In particular, analysis of four $\mathrm{B} \beta$-chain missense mutations found in afibrinogenemic families suggests that normal assembly is not sufficient to insure normal secretion. Using transient transfection assays, Duga et al [15], Spena et al [16], and Vu et al [17] demonstrated that B $\beta$-chains with the substitutions

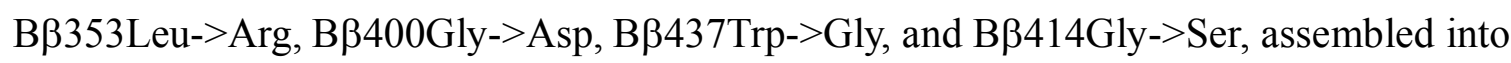
normal 6-chain fibrinogen molecules that were not secreted into media. These data suggest that the $\mathrm{C}$-terminal $\mathrm{B} \beta$-chain domain is critical to fibrinogen secretion but not assembly. Impaired secretion was also apparent in two families with hypofibrinogenemia and hepatic endoplasmic reticulum storage disease, which were examined by Brennan and colleagues. In both cases immunohistologic examination of liver biopsies showed fibrinogen deposition in the endoplasmic reticulum of hepatocytes. In each case a missense mutation was identified in the C-terminal region of the $\gamma$-chain, $\gamma 284 \mathrm{Gly}->\operatorname{Arg}[11]$ and $\gamma 375 \mathrm{Arg}->\operatorname{Trp}[12] . \quad$ The authors suggested that the misfolding of the variant protein caused aggregation within the endoplasmic 
reticulum, leading to hypofibrinogenemia. It is unclear whether the misfolded proteins were fully assembled fibrinogen or assembly intermediates or individual chains, as the hepatic inclusions were not examined. Nevertheless, it is clear that single substitutions within the C-terminal $\gamma$-chain domain cause hypofibrinogenemia. Our analysis of CHO cell lysates showed no accumulation of the variant molecules indicating that aggregation of the variant was not the basis for this hypodysfibrinogenemia.

In summary, the results presented here support the hypothesis that the reduced levels of human $\gamma \Delta 319,320$ fibrinogen in the plasma of Otsu and Vlissingen/Frankfurt-IV families and the reduced levels of mouse $\gamma \Delta 319,320$ fibrinogen in the plasma of targeted mice arises from modestly impaired secretion of this variant fibrinogen.

\section{ACKNOWLEDGEMENTS}

This work was partly supported by a Grant-in-Aid for Science Research from the Ministry of Education, Science and Culture of Japan (No.16590451, NO) and the US National Institutes of Health (R01 HL68836, STL). 


\section{REFERENCES}

1. Blomback B. Fibrinogen and fibrin-proteins with complex roles in hemostasis and thrombosis. Thromb Res 1996;83:1-75.

2. Doolittle RF. Fibrinogen and fibrin. Ann Rev Biochem 1984;53:195-229.

3. Yu S, Sher B, Kudryk B, Redman CM. Fibrinogen precursors. Order of assembly of fibrinogen chains. J BiolChem 1984;259:10574-81.

4. Huang S, Mulvihill ER, Farrell DH, et al. Biosynthesis of human fibrinogen. Subunit interactions and potential intermediates in the assembly. J Biol Chem 1993;268:8919-26.

5. Huang S, Cao Z, Chung DW, et al. The role of $\beta \gamma$ and $\alpha \gamma$ complexes in the assembly of human fibrinogen. J Biol Chem 1996;271:27942-7.

6. Redman CM, Xia H. Fibrinogen biosynthesis. Assembly, intracellular degradation, and association with lipid synthesis and secretion. Ann New York Acad Sci USA 2001;936:480-95.

7. Okumura N, Terasawa F, Tanaka $\mathrm{H}$, et al. Analysis of fibrinogen $\gamma$-chain truncations shows the C-terminus, particularly $\gamma \mathrm{Ile} 387$, is essential for assembly and secretion of this multichain protein. Blood 2002;99:3654-60.

8. http://www.geht.org/databaseang/fibrinogen/

9. Brennan SO, Wyatt JM, May S, et al. Hypofibrinogenemia due to novel 316 Asp->Tyr substitution in the fibrinogen B $\beta$ chain. Thromb Haemost 2001;85:450-3.

10. Terasawa F, Okumura N, Kitano K, et al. Hypofibrinogenemia associated with a heterozygous missense mutation $\gamma 153 \mathrm{Cys}$ to Arg (Matsumoto IV): In vitro expression demonstrates defective secretion of the variant fibrinogen. Blood 1999;94:4122-31.

11. Brennan SO, Wyatt J, Medicina D, et al. Fibrinogen Brescia. Hepatic endoplasmic reticulum storage and hypofibrinogenemia because of a 284 Gly- $>$ Arg mutation. Am J Pathol $2000 ; 157 ; 189-96$.

12. Brennan SO, Maghzal G, Shneider BL, et al. Novel fibrinogen $\gamma 375$ Arg-> Trp mutation (fibrinogen Aguadilla) causes hepatic endoplasmic reticulum storage and hypofibrinogenemia. Hepatology 2002;36;652-8.

13. Brennan SO, Wyatt JM, Fellowes AP, et al. $\gamma 371$ Thr->Ile substitution in the fibrinogen $\gamma D$ domain causes hypofibrinogenaemia. Biochim Biophys Acta 2001;1550:183-8.

14. Maghzal GJ, Brennan SO, Fellows AP, et al. Familial hypofibrinogenemia associated with heterozygous substitution of a conserved arginine residue; B $\beta 255 \mathrm{Arg}->\mathrm{His}$ (fibrinogen Merivale). Biochim Biophys Acta 2003;1645:146-51. 
15. Duga S, Asselta R, Santagostino E, et al. Missense mutations in the $\beta$ fibrinogen gene cause congenital afibrinogenemia by impairing fibrinogen secretion. Blood 2000;95:1336-41.

16. Spena S, Asselta O, Duga S, et al. Congenital afibrinogenemia: intracellular retention of fibrinogen due to a novel W437G mutation in the fibrinogen B $\beta$-chain gene. Biochim Biophys Acta 2003;1639:87-94.

17. Vu D, Bolton-Maggs PHB, Parr JR, et al. Congenital afibrinogenemia: identification and expression of a missense mutation in FGB impairing fibrinogen secretion. Blood 2003;102:4413-5.

18. Terasawa F, Hogan KA, Kani S, et al. Fibrinogen Otsu I: a $\gamma$ Asn319, $\gamma$ Asp320 deletion dysfibrinogen identified in an asymptomatic pregnant woman. Thromb Haemost 2003;90:757-8. 19. Koopman J, Haverkate F, Briet E, et al. A congenitally abnormal fibrinogen (Vlissingen) with a 6-base deletion in the $\gamma$-chain gene, causing defective calcium binding and impaired fibrin polymerization. J Biol Chem 1991;266:13456-61.

20. Hogan KA, Gorkun OV, Lounes KC, et al. Recombinant fibrinogen Vlissingen/Frankfurt IV. The deletion of residues 319 and 320 from the $\gamma$ chain of fibrinogen alters calcium binding, fibrin polymerization, cross-linking, and platelet aggregation. J Biol Chem 2000;275:17778-85.

21. Okumura N, Furihata K, Terasawa F, et al. Fibrinogen Matsumoto II: $\gamma^{308}$ Asn- $>$ Lys (AAT->AAG) mutation associated with bleeding tendency. Br J Haematol 1996;94:526-8. 22. Okumura N, Gorkun OV, Lord ST. Severely impaired polymerization of recombinant fibrinogen $\gamma$-364 Asp-> His, the substitution discovered in a heterozygous individual. J Biol Chem 1997;272:29596-601.

23. Mihalyi E. Physicochemical studies of bovine fibrinogen. IV. Ultraviolet absorption and its relation to the structure of the molecule. Biochemistry. 1968;7:208-23.

24. Hogan KA, Lord ST, Okumura N, et al. A functional assay suggests that heterodimers exist in two C-terminal $\gamma$-chain dysfibrinogens: Matsumoto I and Vlissingen/Frankfurt IV. Thromb Haemost 2000;83:592-7.

25. Binnie CG, Hettasch JM, Strickland E, et al. Characterization of purified recombinant fibrinogen: Partial phosphorylation of fibrinopeptide A. Biochemistry 1993;32:107-13. 26. Hogan KA, Merenbloom BK, Kim HS, et al. Neonatal bleeding and decreased plasma fibrinogen levels in mice modeled after the dysfibrinogen Vlissingen/Frankfurt IV. J Thromb Haemost 2004;2:1484-7. 


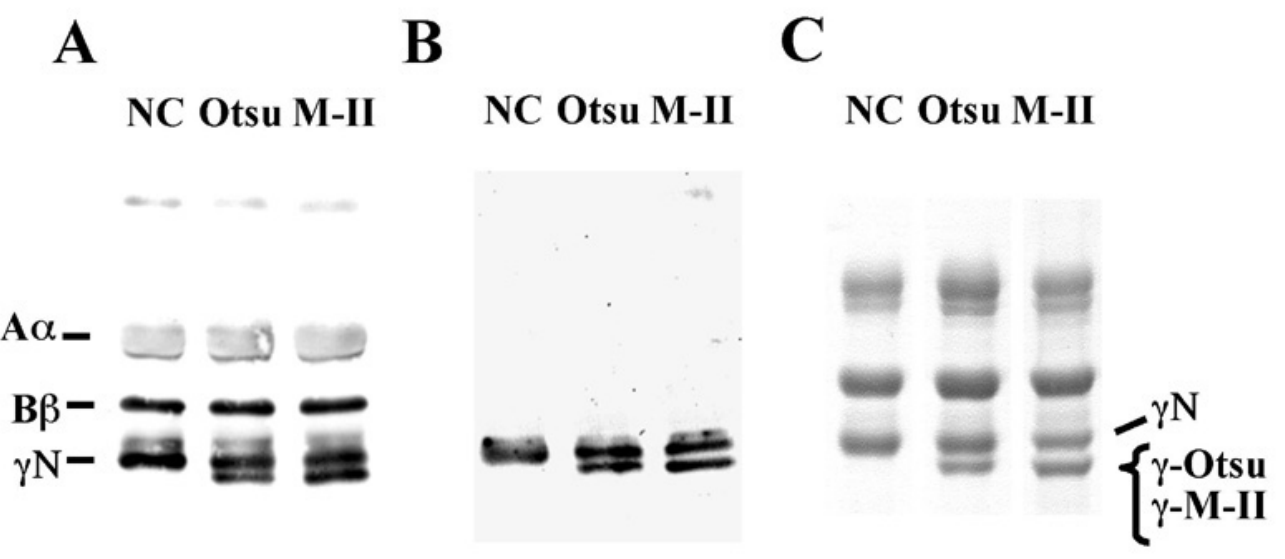

Figure 1. SDS-PAGE and immunoblot analysis of plasma and purified fibrinogen.

Samples of diluted plasma (100 ng of fibrinogen) (A, B) or purified fibrinogen (3 $\mu \mathrm{g})(\mathrm{C})$ were subjected to $10 \%$ SDS-PAGE under reduced conditions. Blots were developed with an anti-fibrinogen polyclonal antibody (A) or an anti- $\gamma$-chain monoclonal antibody (4A5) (B), as described in methods. Reacting bands were detected by alkaline phosphatase-catalyzed color development (A, B). SDS-PAGE gels were stained with Coomassie Brilliant Blue R-250 (C). Lane NC: normal control, lane Otsu: fibrinogen Otsu, and lane M-II: fibrinogen Matsumoto II $(\gamma 308 \mathrm{~K})$. 

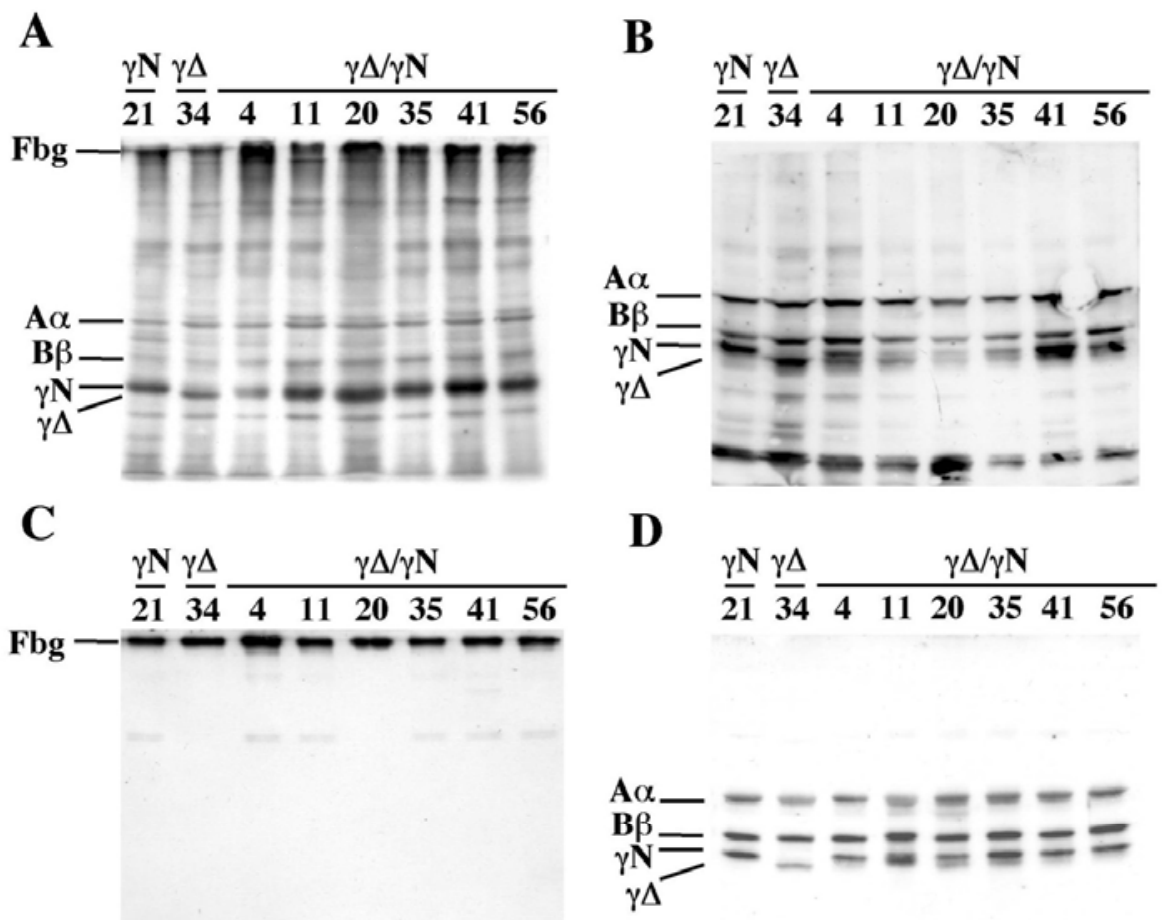

Figure 2. Western blot analysis of the $\mathrm{CHO}$ cell lysates and culture media. CHO cell lysates ( $5 \mathrm{ng}$ of fibrinogen) were subjected to $8 \%$ SDS-PAGE under nonreduced (A) or reduced conditions (B). The samples of culture media (5 ng of fibrinogen) were subjected to $8 \%$ SDS-PAGE under nonreduced (C) or reduced conditions (D). Blots were developed with an anti-fibrinogen polyclonal antibody as described in methods. Reacting bands were detected by chemiluminescence. 


\section{A: $\gamma$ N21-CHO cells}
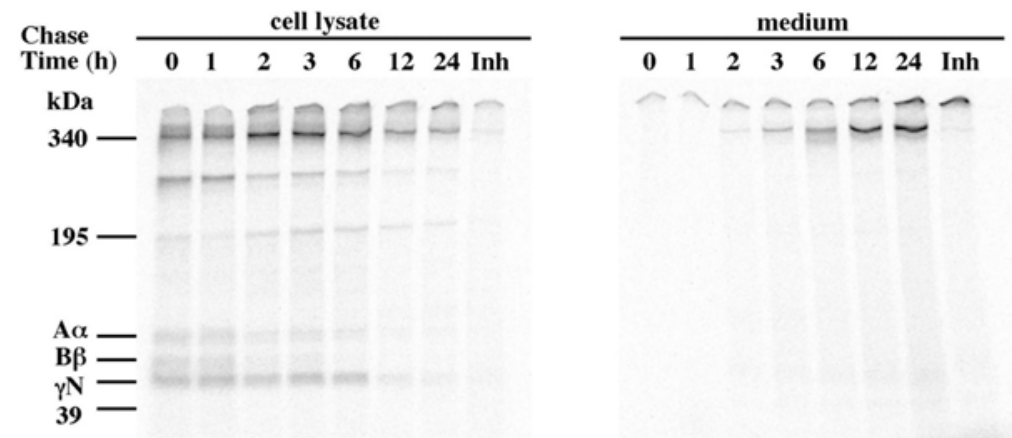

\section{B: $\gamma \Delta 34-C H O$ cells}
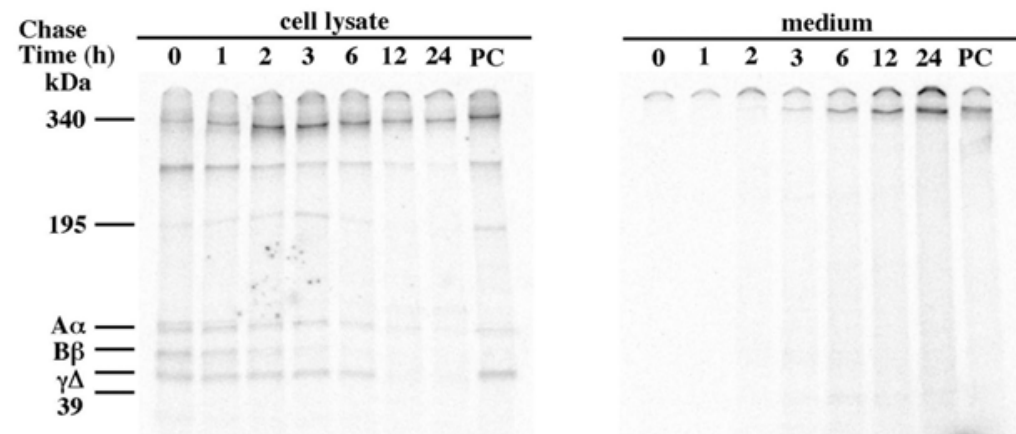

\section{C: $\gamma \Delta / \gamma$ N11-CHO cells}
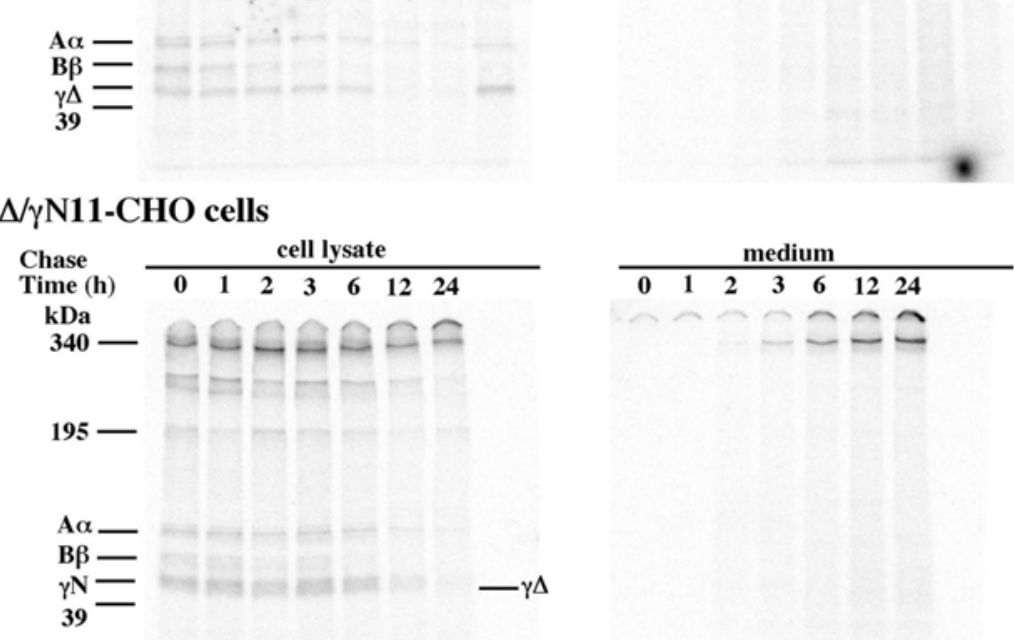

Figure 3. Analysis of pulse-labeled fibrinogen in the transfected $\mathrm{CHO}$ cells. Cells were labeled for 1 hour with [35S]-methionine and chased for the indicated periods with an excess of unlabeled methionine. Immunoprecipitates from the cell lysates or conditioned media were analyzed on $4 \%$ to $12 \%$ gradient SDS-PAGE under nonreducing conditions and labeled bands detected by autoradiography. Lane Inh included the addition of purified plasma fibrinogen to the reaction mixtures of the 6-hour chase experiment to demonstrate the antibody specificity. Lane $\mathrm{PC}$ in normal media was the 2 -fold diluted conditioned medium at the 12 -hour chase of $\gamma \mathrm{N}-\mathrm{CHO}$ cells as a positive control. 


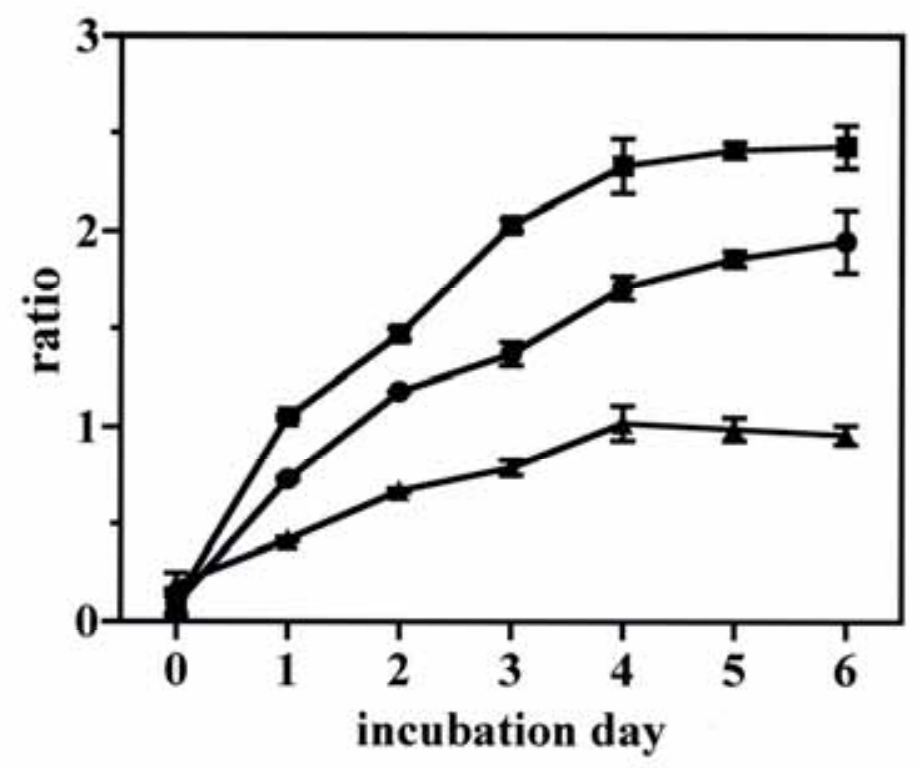

Figure 4. Secretion of fibrinogen from confluent $\mathrm{CHO}$ cells. Fibrinogen concentrations were determined by ELISA every 24 hrs for 6 days. The ratio of the fibrinogen concentrations in the media determined at the indicated times/the mean concentration in the respective cell lysate at day 0 , for $\gamma \mathrm{N}-\mathrm{CHO}$ cells $(\boldsymbol{\square}), \gamma \Delta$-CHO cells $(\boldsymbol{\Delta})$, and $\gamma \Delta / \gamma \mathrm{N}-\mathrm{CHO}$ cells $(\mathbf{O})$ was plotted. Results represent the mean \pm SD of the triplicate cultures. 